ARTICLE

https://doi.org/10.1038/s41467-019-08749-2

\title{
On the degradation mechanisms of quantum-dot light-emitting diodes
}

Song Chen ${ }^{1}$, Weiran Cao ${ }^{2}$, Taili Liu ${ }^{3}$, Sai-Wing Tsang ${ }^{3}$, Yixing Yang ${ }^{2}$, Xiaolin Yan $^{2}$ \& Lei Qian ${ }^{2}$

The operating lifetime of blue quantum-dot light-emitting diodes (QLED) is currently a short slab for this emerging display technology. To pinpoint the origin of device degradation, here we apply multiple techniques to monitor the electric-field distribution and space-charge accumulation across the multilayered structure before and after lifetime tests. Evident by charge-modulated electro-absorption and capacitance-voltage characteristics, the excited electrons in blue quantum dots (QD) are prone to cross the type II junction between the QD emission layer and the electron-transporting layer (ETL) due to the offset of conduction band minimum, leading to space-charge accumulation and operating-voltage rise in the ETL. Therefore, unlike those very stable red devices, of which the lifetime is primarily limited by the slow degradation of hole-transporting layer, the poor lifetime of blue QLED originates from the fast degradation at the QD-ETL junction. Materials engineering for efficient electron injection is prerequisite for the boost of operating lifetime.

\footnotetext{
${ }^{1}$ College of Chemistry, Chemical Engineering and Materials Science, Soochow University, 199 Ren'ai Road, Suzhou Industrial Park, Suzhou 215123 Jiangsu China. ${ }^{2}$ TCL Corporate Research, 1001 Zhongshan Park Road, Nanshan District, Shenzhen 518067 Guangdong, China. ${ }^{3}$ Department of Materials Science and Engineering, City University of Hong Kong, 83 Tat Chee Avenue, Kowloon Tong, Hong Kong SAR, China. These authors contributed equally: Song Chen, Weiran Cao. Correspondence and requests for materials should be addressed to S.C. (email: songchen@suda.edu.cn) or to L.Q. (email: qianlei@tcl.com)
} 
C ombining the features of light-emitting quantum dots and solution processing, QLED has become an emerging display technology potentially capable of $100 \%$ Rec. 2020 color gamut, high luminance efficiency and low-cost manufacturing ${ }^{1-3}$. Thanks to the development of quantum-dot synthesis $^{4-7}$ and device architectures ${ }^{8-10}$, lab-scale devices with external quantum efficiency from 10 to $20 \%$ and emission FWHM $\sim 25 \mathrm{~nm}$ have been demonstrated for all the three primary colors for full-color display ${ }^{10-12}$. Moreover, since the technology is highly compatible with printed display, growing number of display manufacturers have included QLED in their roadmaps ${ }^{13}$.

Despite of these achievements, QLED is still in the early stage of research and development. To productize this technology, researchers have to solve the issue of device degradation. Based upon published materials, red, green and blue QLED devices exhibit greatly different operating lifetime, therefore they do not share a universal degradation mechanism. For the ease of comparison, the following referred data are converted to LT50, the time at which the intensity of electroluminescence decays to $50 \%$ of its initial value $-L_{0}=1000 \mathrm{~cd} \mathrm{~m}^{-2}$, using the raw data and provided acceleration factors. As reported, the lifetime of red QLED has well exceeded $3000 \mathrm{~h}^{11,12,14,15}$; green devices are behind but still capable of $1000 \mathrm{~h}^{14}$; blue QLED is the worst among the three, delivering lifetime just over $20 \mathrm{~h}^{12,14,16}$. Comparing with a rival technology, e.g., printed organic light-emitting diodes (OLED), QLED is far behind because inkjet-printed OLED have reached LT95 $\left(L_{0}=1000 \mathrm{~cd} \mathrm{~m}^{-2}\right)$ of $7000 \mathrm{~h}$ for red, $9000 \mathrm{~h}$ for green, and $500 \mathrm{~h}$ for blue ${ }^{17,18}$.

QLED devices have been using device structures developed for OLED, but their degradation mechanism is worth separated study due to the difference in materials. Unlike organic emitters, the ionization potentials and electron affinities of light-emitting QDs cannot be consistently characterized by existing methods ${ }^{10,19,20}$. Photo-electron spectroscopy, with an analysis depth about $1 \mathrm{~nm}^{21}$, has difficulty in resolving the electronic structure of QDs with core-shell or gradient-alloyed nanostructures. Currently, QLED devices are mostly formed with a structure of four functional layers plus a pair of electrodes. The highest occupied molecular orbitals (HOMOs) of hole-transporting layer (HTL) materials, which were designed for OLED, cannot match the deep-lying valence-band maximum (VBM) of Cd-containing QDs. Besides that, the effect of hole mobility and material stability should be considered. For example, poly(9-vinlycarbazole) (PVK, hole mobility $\left.\mu_{\mathrm{h}} \sim 10^{-6} \mathrm{~cm}^{2} \mathrm{~V}^{-1} \mathrm{~s}^{-1}\right)^{22}$ is widely used in blue devices for high quantum efficiency ${ }^{12,23}$, however, due to the very low carrier mobility and the instability of $\mathrm{C}-\mathrm{N}$ bond ${ }^{24}$, it is known for the negative impact on operating lifetime ${ }^{23}$. In contrast, poly (9,9-dioctylfluorene-co- $N$-(4-(3-methylpropyl))diphenylamine) (TFB) has better stability and hole mobility $\left(\mu_{\mathrm{h}}>10^{-4} \mathrm{~cm}^{2} \mathrm{~V}^{-1}\right.$ $\left.\mathrm{s}^{-1}\right)$. Currently, the best lifetime of blue QLED is achieved using TFB as HTL despite of the mismatch of VBM ${ }^{14,16}$. Electrontransporting layers (ETL) are commonly made of zinc oxide nanoparticles $(\mathrm{NPs})^{9-11}$. Based upon the reported data, $\mathrm{ZnO}$ NPs can efficiently inject electrons into the red QDs shelled by $\mathrm{CdS}^{10,11}$ or composed of a gradient-alloyed structure $\left(\mathrm{Cd}_{1-\mathrm{x}} \mathrm{Zn}_{\mathrm{x}} \mathrm{Se}_{1-\mathrm{y}} \mathrm{S}_{\mathrm{y}}\right)^{9,12}$. For green and blue QDs, which are commonly terminated with $\mathrm{ZnS}$ at surface, the electronic property is less reported. Without experimental data, reported band diagrams cannot agree on whether an Ohmic or Schottky contact is formed between $\mathrm{ZnO}$ and $\mathrm{QDs}{ }^{25-27}$. As the electron affinity decreases in the sequence of red, green and blue $\mathrm{QDs}^{28}$, the injection of electron may be a major difference between blue and red devices. So far, reports of QLED mostly cover the topics of luminescence efficiency, lifetime values, and printing methods. To our best knowledge, experimental study on the degradation mechanisms is very limited.
To study the degradation mechanisms, here we choose a popular device structure composed of a transparent anode, a polymeric hole-injection layer (HIL), a polymeric HTL, an emission layer (EML) assembled of red $\left(\mathrm{Cd}_{1-\mathrm{x}} \mathrm{Zn}_{\mathrm{x}} \mathrm{Se}_{1-\mathrm{y}} \mathrm{S}_{\mathrm{y}}\right)$ or blue $\left(\mathrm{Cd}_{1-\mathrm{x}} \mathrm{Zn}_{\mathrm{x}} \mathrm{S}\right)$ dots, an ETL assembled of $\mathrm{ZnO} \mathrm{NPs}$, and a top cathode. We choose to focus on blue devices because it is currently the short slab of the full-color QLED display. Red devices are chosen as controls because they outperform the blue ones by more than 100 times. In particular, we choose red $\left(\mathrm{Cd}_{1-\mathrm{x}} \mathrm{Zn}_{\mathrm{x}} \mathrm{Se}_{1-\mathrm{y}} \mathrm{S}_{\mathrm{y}}\right)$ and blue $\left(\mathrm{Cd}_{1-\mathrm{x}} \mathrm{Zn}_{\mathrm{x}} \mathrm{S}\right)$ QDs with a composition gradient from core to surface ( $\mathrm{x}$, $\mathrm{y}$ increases) which enables highly efficient electroluminescence for all the red, green and blue devices ${ }^{12}$. As blue QDs are chemically similar to those very stable red QDs (Supplementary Fig. 1d), here we assume the short lifetime of blue devices is not primarily due to the chemical instability of QDs.

Red and blue devices will be compared side by side throughout the following section. We begin with the results of lifetime tests. To determine the source of degradation in blue QLED, we then analyze electro-absorption (EA) spectra for the organic HTL, the QD emission layer and the ETL to spot possible space-charge accumulation. Finally we combined the results of EA and capacitance-voltage $(C-V)$ characteristics to determine the field distribution across the whole device. Unexpectedly, the slightly degraded HTL, in which oxidized molecules $\left(\mathrm{HTL}^{+}\right)$are spotted, is barely responsible for device degradation. On the contrary, the $\mathrm{ZnO}$-based ETL is concluded as the major source of operatingvoltage rise due to the charge transfer across the QD-ZnO junction and charge accumulation in the ETL. Although the result appears counter-intuitive since oxides are usually more stable than organic materials, the offset of conduction band maximum (CBM) across the $\mathrm{ZnO}-\mathrm{QD}$ junction causes the unwanted charge transfer.

\section{Results}

Device performance. Figure 1 shows the result of lifetime tests. Blue devices with TFB HTL show LT50 $\left(L_{0}=1000 \mathrm{~cd} \mathrm{~m}^{-2}\right)$ of $23 \mathrm{~h}$ which make sure the devices do not significantly degrade during our EA and $C-V$ measurements. As mentioned, the poor lifetime of PVK device (LT50 2 h, $L_{0}=1000 \mathrm{~cd} \mathrm{~m}^{-2}$ ) is attributed to the instability of PVK. In comparison, the red device with TFB HTL shows a lifetime (LT90, $L_{0}=1000 \mathrm{~cd} \mathrm{~m}^{-2}$ ) more than $800 \mathrm{~h}$, indicating the primary degradation mechanism is different between red and blue devices. Other regular characterization results of QLED are summarized in Supplementary Fig. 1.

Despite of the same device structure, red and blue QDs have different $\mathrm{CBM}$ and VBM values. Figure 1c shows the $J-F$ characteristics of electron-only devices. Obviously, the sample with red dots has much higher electron current than the sample with blue dots, indicating a large difference of electron-injection barrier. In contrast, the hole injection from TFB to QDs does not follow the same trend (Supplementary Fig. 1c). These results suggest the charge balance in blue devices is poor, but they cannot accurately reflect the situation in a real QLED device. In the following, we decide to apply more powerful techniques to monitor each functional layer of red and blue devices before and after lifetime tests.

Hole-transporting layer. From the study of OLED, we learned that a hole-injection barrier results in space-charge accumulation and operating-voltage rise in organic $\mathrm{HTLs}^{29,30}$. Due to the large ionization potentials, the VBM of Cd-containing QDs cannot be matched by the HOMOs of the HTL materials designed for OLED, therefore the HTL is a possible source of degradation. In this part, we choose to first discuss TFB which currently enables competitive lifetime results $\left(\mathrm{LT} 50 \sim 23 \mathrm{~h}, L_{0}=1000 \mathrm{~cd} \mathrm{~m}^{-2}\right.$, 

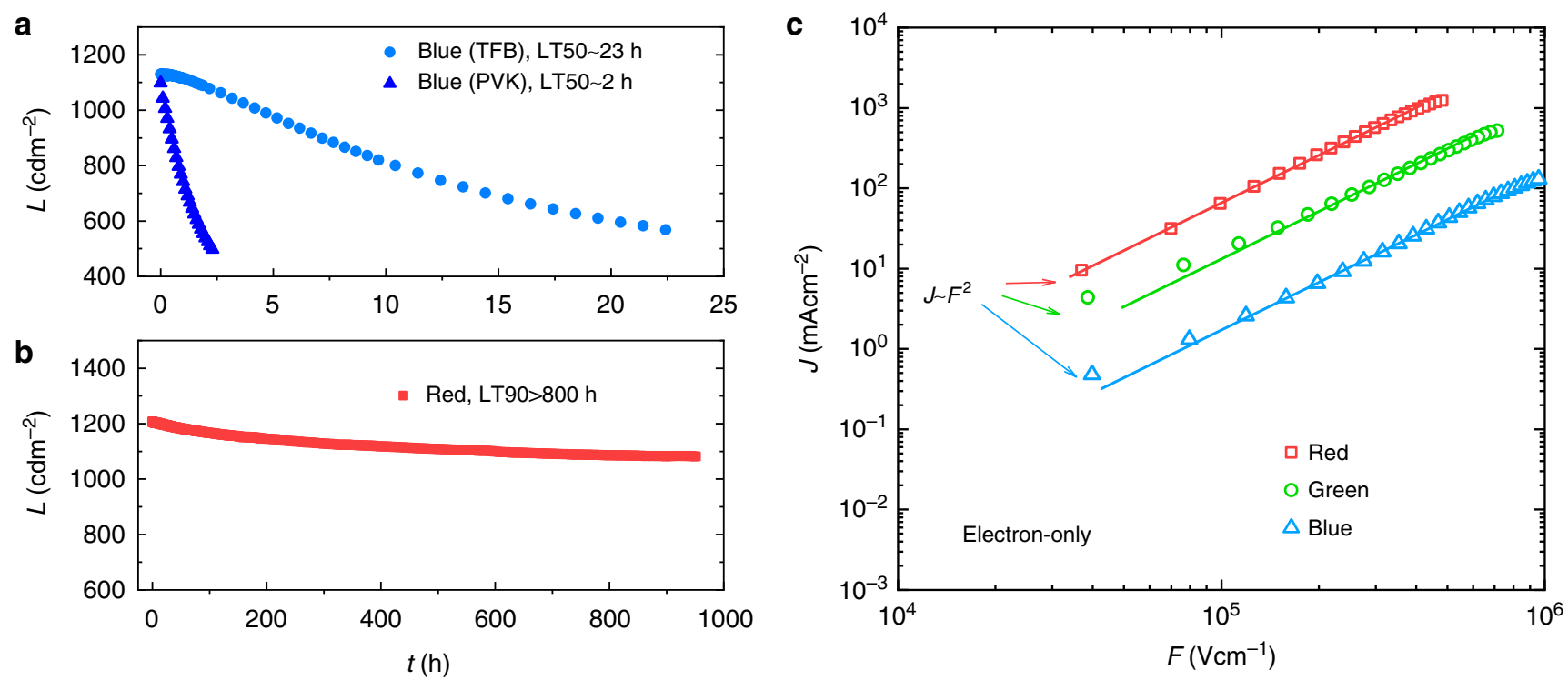

Fig. 1 Comparing red and blue devices. a Lifetime test results of blue devices. b Lifetime results of red devices. c $J-F$ characteristics of electron-only samples. These electron-only devices have a structure of ITO/ZnO/QDs/ZnO/AI

Fig. 1a) ${ }^{16}$. The discussion of another commonly used HTL (PVK) is presented in supplementary information.

To study HTL, we measured EA spectra for red and blue QLED devices before and after lifetime tests. As seen in Fig. 1b, the red devices made and tested in the same batch exhibit LT90 $\left(L_{0}=\right.$ $1000 \mathrm{~cd} \mathrm{~m}^{-2}$ ) over $800 \mathrm{~h}$. In the EA test, the sample was placed under reversed bias to avoid charge injection. As shown in Fig. 2a, the electro-absorption of TFB ranges from 400 to $450 \mathrm{~nm}$. The signal at longer wavelengths is from red QDs. Comparing the LT100 and LT90 device, we find them resemble very similar spectra except the TFB region, which will later be attributed to the emergence of $\mathrm{TFB}^{+}$. In Fig. 3a, the in-phase signal scales linearly with the applied DC bias, suggesting a typical Stark effect of TFB which is described as

$$
\begin{aligned}
& \Delta \alpha(\lambda)=-\frac{1}{d} \cdot \frac{\Delta T(\lambda)}{T(\lambda)} \propto \operatorname{Im} \chi^{(3)} \cdot[E(t)]^{2} \\
= & \operatorname{Im} \chi^{(3)} \cdot\left(2 E_{\mathrm{dc}} E_{\mathrm{ac}} \sin \omega t+\frac{1}{2} E_{\mathrm{ac}}^{2} \cos 2 \omega t\right)
\end{aligned}
$$

wherein $\lambda$ is the wavelength of the probing light; $\alpha$ is the absorption coefficient; $T$ is the transmission; $\Delta T$ is change of transmission due to electric field; $\chi^{(3)}$ is the imaginary part of the third-order susceptibility of the probed material ${ }^{31}$. A sinusoidal reference field $\left(E_{\mathrm{ac}} \cos \omega t\right)$ is superimposed upon the DC bias $\left(E_{\mathrm{dc}}\right)$ for modulation and low-noise detection.

As seen in Fig. 1a, the blue devices made and tested in the same batch show LT90 $\left(L_{0}=1000 \mathrm{~cd} \mathrm{~m}^{-2}\right)$ around $4.5 \mathrm{~h}$ and LT50 $\left(L_{0}=1000 \mathrm{~cd} \mathrm{~m}^{-2}\right)$ of $23 \mathrm{~h}$. Figures $2 \mathrm{~b}$ and $3 \mathrm{~b}$, respectively, shows the EA spectra and its bias dependence (wavelength fixed at $415 \mathrm{~nm}$ ) of blue QLED devices. The EA signal from blue QDs ranges from 430 to $470 \mathrm{~nm}$ which is the energy of optical bandgap. Similar to what observed in the degraded red QLED device, the in-phase signal from TFB, $415 \mathrm{~nm}$ in this case, is relatively strengthened, resulting in a red-shift of this feature. Spectrum change like this has been reported for small molecule OLED wherein a portion of HTL molecules are permanently oxidized $\left(\mathrm{HTL}^{+}\right)$after lifetime test due to the non-Ohmic contact between HIL and HTL $^{29}$. Since the optical transition energy of $\mathrm{HTL}^{+}$is smaller that of the corresponding HTL molecule, the resultant EA spectrum appears to be red shifted comparing to

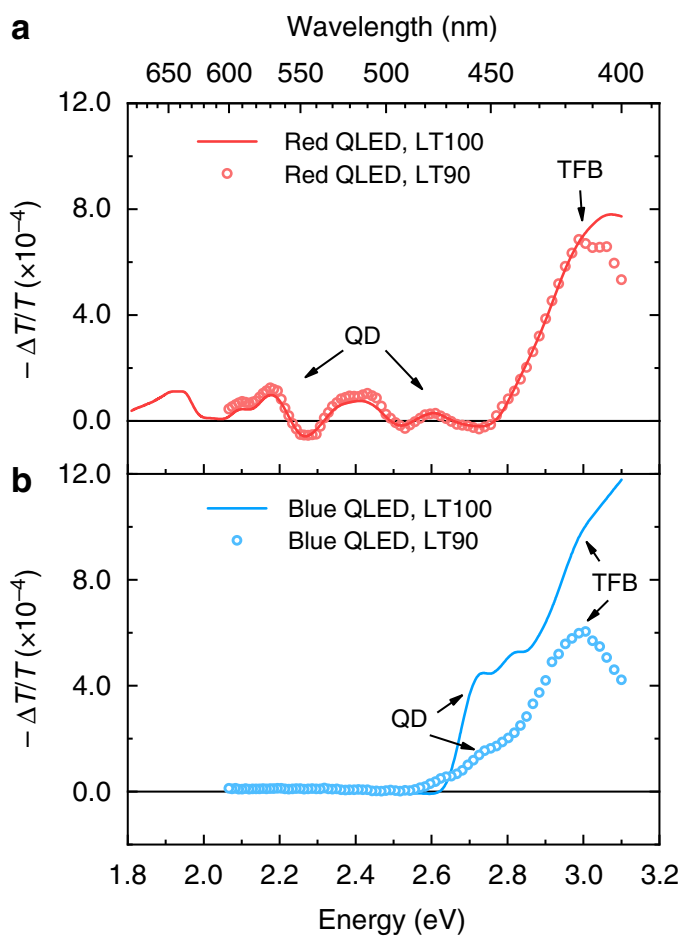

Fig. 2 Electro-absorption spectra. a In-phase spectra of standard red devices (ITO/PEDOT:PSS/TFB/red quantum dots (QD)/ZnO/Al). b Inphase spectra of standard blue devices (ITO/PEDOT:PSS/TFB/blue QD/ $\mathrm{ZnO} / \mathrm{Al}$ ). The measurements are carried out using a $\mathrm{DC}$ bias of $-1 \mathrm{~V}, \mathrm{AC}$ amplitude of $0.1 \mathrm{~V}$. For each device, the spectrum of electro-absorption is taken before and after the lifetime test. Arrows mark contribution from the hole-transporting layer and QD

that of a LT100 sample. In our test, the concentration of spacecharge is modulated by the reference bias $V_{\mathrm{ac}}$ and therefore it contributes charge-modulated signals in addition to the Stark shift $^{29,32,33}$ :

$$
\Delta \alpha=-\frac{1}{d} \cdot \frac{\Delta T}{T}=n \cdot \sigma
$$




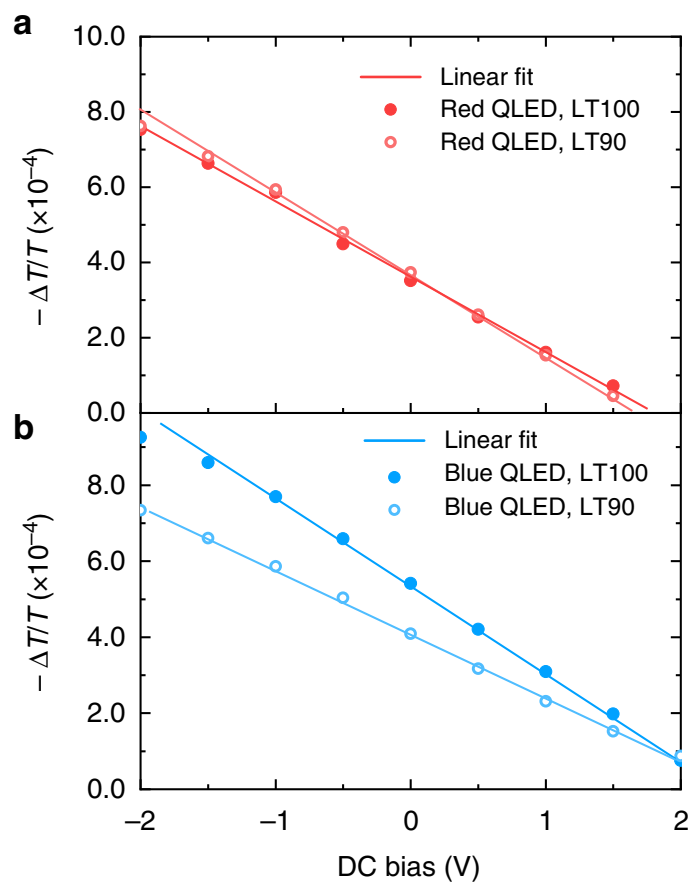

Fig. 3 Electric field across the hole-transporting layer. a Bias-dependent electro-absorption signals of TFB in standard red devices. b Bias-dependent electro-absorption signals of TFB in standard blue devices. The probing wavelength is fixed at $415 \mathrm{~nm}$ for TFB; The DC bias determines the internal electric field. Each device is measured before and after the lifetime test

wherein $n$ is the concentration; $\sigma$ is the absorption cross section of the charged species. Per Eq. 2, the charge-modulated signal is proportional to $n$, thus a heavily degraded HTL layer may exhibit a very different spectrum from that of a fresh sample. Also, since space charges cannot stay in-phase with the modulating bias$V_{\text {ac }}$, the growth of quadrature signal and a non-linear voltage dependence should occur simultaneously. Reviewing the data in Fig. 2, we find that although the emergence of $\mathrm{TFB}^{+}$can be spotted, the quadrature signal due to TFB degradation is negligible (see Supplementary Fig. 2 and the arrowed part in Supplementary Fig. 4a). Therefore, the in-phase signal from TFB remains linear as a function of applied bias (Fig. 3a, b), suggesting typical Stark effect with negligible charge-modulation.

In addition to spectra analysis, the signal strength of the Stark effect can be used to study the electric-field distribution across the multilayered devices ${ }^{34}$. As shown in Figs $2 \mathrm{a}$ and $3 \mathrm{a}$, the signal strength of TFB's Stark effect increases slightly after the LT90 test for the red device, suggesting small voltage rise across the HTL. Per Eq. 1, the contribution of $E_{\mathrm{dc}}$ is in the 1st harmonic portion. When the HTL reaches flat-band condition, the voltage consumption increases gently from $\sim 1.6$ to $\sim 1.7 \mathrm{~V}$ after the LT90 test, suggesting mild degradation in the HTL. In comparison, as shown in Figs $2 \mathrm{~b}$ and $3 \mathrm{~b}$, the field distribution in the HTL changes differently after the LT90 test. First, TFB's signal strength decreases significantly after the LT90 test of the blue device, indicating reduced voltage consumption across the HTL. For a degraded blue QLED device, such voltage drop in the HTL has to be over-compensated by the voltage rise across other functional layers, i.e., the QD EML and the ZnO ETL, because the operating-voltage of the whole device increases significantly. Second, as marked by the intercept on the $x$-axis, the voltage bias at which the HTL reaches flat-band condition is almost unchanged despite of the fast degradation, again confirming that the HTL is barely degraded after the LT90 test.
The result is also consistent with our finding that the hole injection from TFB to red and blue QDs is not significantly different (Supplementary Fig. 1c).

Combining Figs 2 and 3, the emergence of $\mathrm{TFB}^{+}$in the HTL can explain the slow degradation (LT90 $>800 \mathrm{~h}$ ) of red QLED devices. Further effort to improve the lifetime of red QLED should focus on the HTL-QD junction ${ }^{15}$. For the blue QLED, the conclusion depends on specific HTL materials. When the HTL is made of PVK, although the Stark shift of PVK $\left(E_{\mathrm{g}} \sim 3.6 \mathrm{eV}\right)$ is out of the detection range (Supplementary Fig. $4 \mathrm{~b}$ and $\mathrm{c}$ ), the incorporation of PVK reduces the operating lifetime (LT50 $2 \mathrm{~h}$, Fig. 1a). When TFB is used instead, LT50 is improved to $23 \mathrm{~h}$. Nevertheless, our results show that the luminance decay is not primarily determined by the degradation of HTL. As to be shown in the next part, other degradation mechanisms should be considered.

Electron-transporting layer and emission layer. $\mathrm{ZnO}$ has a bandgap energy over $3.4 \mathrm{eV}$, which is greater than our limit $(3.1 \mathrm{eV})$ for low-noise detection, therefore a direct measurement of $\mathrm{ZnO}$ 's Stark shift cannot be done. In addition, the minimum-required photon energy for persistent electron photoconductivity (PPC, or persistent increase of capacitance) of $\mathrm{ZnO}$ is reported to be $3.1 \mathrm{eV}^{35}$, therefore charge-modulated signal due to PPC effect is not expected either. We decided circumvent the issue by measuring the EA response of QDs and finishing the analysis using capacitancevoltage characteristics. To conclude the effect of $\mathrm{ZnO}$ using EA experiments, we included not only QLED devices with regular structures, but also two sets of specially designed samples-one with a pair of electrodes sandwiching a single layer of (red or blue) QDs and the other with electrodes sandwiching a (red or blue) QD layer plus an additional layer of $\mathrm{ZnO}$.

The EA spectra of red $\left(\mathrm{Cd}_{1-\mathrm{x}} \mathrm{Zn}_{\mathrm{x}} \mathrm{Se}_{1-\mathrm{y}} \mathrm{S}_{\mathrm{y}}\right)$ QDs without and with the addition of a $\mathrm{ZnO}$ ETL are plotted in Fig. $4 \mathrm{a}, \mathrm{b}$, respectively. Similar to single-compound QDs, the feature at the optical bandgap (1st excitonic level) is due to the Stark shift of QDs ${ }^{36,37}$. As detailed in supporting materials (Supplementary Fig. 5), additional oscillating features at shorter wavelength are attributed to higher excitonic levels ${ }^{38-40}$. As seen, the incorporation of $\mathrm{ZnO}$ does not change the profile of in-phase spectra. The difference in the signal strength is due to the increase of built-in potential $\left(V_{\mathrm{bi}}\right)$. For both samples, the quadrature signal is negligible throughout the spectra, indicating that the junction between red $\left(\mathrm{Cd}_{1-\mathrm{x}} \mathrm{Zn}_{\mathrm{x}} \mathrm{Se}_{1-\mathrm{y}} \mathrm{S}_{\mathrm{y}}\right)$ QDs and $\mathrm{ZnO}$ cannot generate charges for electric-field modulation. With the probing wavelength fixed at $570 \mathrm{~nm}$, at which the feature is attributed to Stark shift, the bias-dependent in-phase signal is plotted in Supplementary Fig. 3. The amplitude scales linearly with the applied bias, suggesting negligible charge-modulation effect.

The EA spectra of blue $\left(\mathrm{Cd}_{1-\mathrm{x}} \mathrm{Zn}_{\mathrm{x}} \mathrm{S}\right)$ QDs without and with an additional ZnO ETL are plotted in Fig. 4c, d, respectively. Around the optical gap energy of blue $\left(\mathrm{Cd}_{1-\mathrm{x}} \mathrm{Zn}_{\mathrm{x}} \mathrm{S}\right)$ QDs, two peaks can be resolved in both figures. Comparing to the samples of red QDs, the in-phase signals also increase with the addition of $\mathrm{ZnO}$, however, the EA spectra show very different characteristics. Without the $\mathrm{ZnO}$ layer (Fig. 4c), the response of blue QDs is in-phase with the modulating field-showing negligible quadrature signal. With the addition of a $\mathrm{ZnO}$ layer (Fig. 4d), the quadrature signal around the optical bandgap grows by more than an order of magnitude due to the charge-modulation effect which is discussed later. The bias dependence measurement at given probing wavelength was further carried out to confirm the result of the spectra analysis. Without $\mathrm{ZnO}$ (Fig. 5a), the linear relationship between the in-phase signal and applied bias is maintained with a probe beam at the wavelength of $455 \mathrm{~nm}$, indicating that the EA response is dominated by the effect of Stark shift. With the addition of $\mathrm{ZnO}$ (Fig. 5b), the $-\Delta T / T$ 


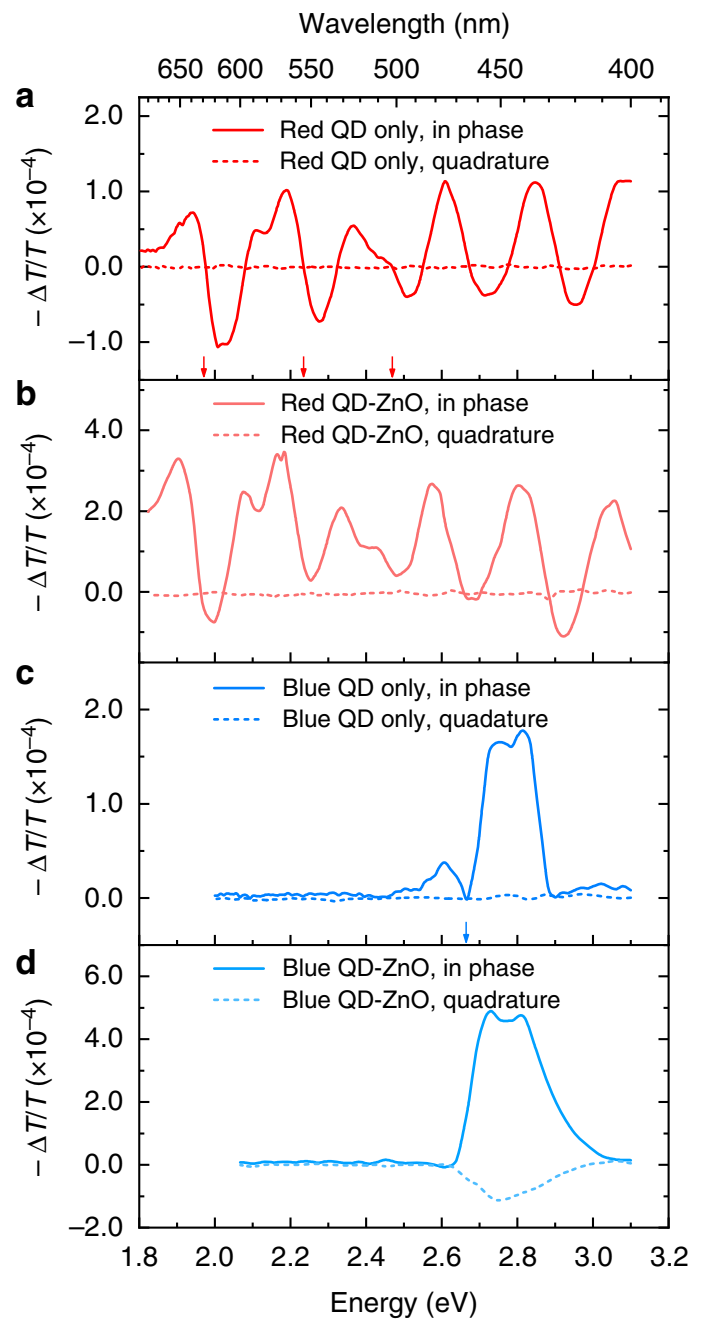

Fig. 4 The quantum-dot-ZnO junction. a Electro-absorption (EA) spectra (in-phase and quadrature) of the sample with a structure of ITO/red $\left(\mathrm{Cd}_{1-x} \mathrm{Zn}_{x} \mathrm{Se}_{1-y} \mathrm{~S}_{y}\right)$ quantum dots (QDs)/Al. b EA spectra (in-phase and quadrature) of the sample with a structure of ITO/red $\left(\mathrm{Cd}_{1-x} \mathrm{Zn}_{x} \mathrm{Se}_{1-y} \mathrm{~S}_{y}\right)$ $\mathrm{QDs} / \mathrm{ZnO} / \mathrm{Al}$. c EA spectra (in-phase and quadrature) of the sample with a structure of ITO/blue $\left(\mathrm{Cd}_{1_{-x}} \mathrm{Zn} \mathrm{n}_{x} \mathrm{~S}\right)$ QDs/AI. d EA spectra (in-phase and quadrature) of the sample with a structure of ITO/blue $\left(\mathrm{Cd}_{1-x} \mathrm{Zn} \mathrm{n}_{\mathrm{x}} \mathrm{S}\right) \mathrm{QDs} /$ $\mathrm{ZnO} / \mathrm{Al}$. The spectra are obtained using a DC bias of $-1 \mathrm{~V}, \mathrm{AC}$ amplitude of $0.1 \mathrm{~V}$. Arrows mark the wavelength at which in-phase signal reaches zero and they also match excitonic peaks in linear spectra (Supplementary Fig. 1). Relevant discussion is detailed in Supplementary Note 4

vs. bias relationship is completely irrelevant to Eq. 1. Instead, both in-phase and quadrature signals (at $455 \mathrm{~nm}$ ) resemble characteristics of a leaky capacitor wherein the amount of charges for field modulation reduces when $E$ field increases. Therefore, the addition of a $\mathrm{ZnO}$ layer to blue QDs introduces strong charge modulation effect.

The contrasting effect of $\mathrm{ZnO}$ ETL on red and blue QDs is also observed in standard QLED devices. First, as seen in the spectra of Supplementary Fig. 2, red devices show no sign of quadrature signals before or after the lifetime tests, while blue devices with either TFB or PVK HTLs (Supplementary Fig. 4a and c) shows strong quadrature spectra around the wavelength of $460 \mathrm{~nm}$, which is consistent with Fig. 4d. Second, as shown in Fig. 5c, d, the bias dependence of QDs' EA signal is measured for LT100 and degraded QLED devices. For the red QLED devices after the LT90 test (Fig. 5c), the signal strength of red QDs increases slightly comparing to that of the LT100 control device. Similar to the observation from Fig. 3a, the voltage across the red QD layer does not increase significantly during degradation, which is consistent with the result that red devices experience marginal operating-voltage rise after the LT90 test. The linear $-\Delta T / T$ vs. bias relationship and negligible quadrature signal confirms there is no charge modulation across the QD-ZnO junction in red QLED devices. In Fig. 5d, the bias dependence of in-phase and quadrature signal is consistent with that in Fig. 5b, demonstrating strong charge modulation in the standard blue QLED device at both negative and positive bias. The corresponding spectra taken at $V_{\mathrm{dc}}=-1 \mathrm{~V}$ are shown in Supplementary Fig. 4a. Here, we need to point out that, although the charge modulated signal is linked with device degradation, the transfer function from | $-\Delta T / T_{\text {quadrature }}$ to the luminance degradation cannot be established at this stage because the amount of charges available for field modulation is cumulatively determined by the internal electric field across the QD-ZnO junction and the generation of charged species. In heavily degraded samples, the internal field at given bias increases significantly due to the increased operatingvoltage. As a result, the $-\Delta T / T$ vs. bias curve is translated along the voltage axis by +2 to $+3 \mathrm{~V}$ (Fig. $5 \mathrm{~d}$ ), which is consistent with the operating-voltage rise during the lifetime test, leading to reduction of quadrature signal at given bias.

Summarizing the results of Figs 4 and 5 into Supplementary Table 1, we notice that the charge modulation features, either in the spectra (Fig. 4d) or bias dependence plot (Fig. 5b, d), only occur to the samples with both blue QDs and $\mathrm{ZnO}$ ETL. To explain the origin of charge modulation, electron-transfer from blue $\left(\mathrm{Cd}_{1-\mathrm{x}} \mathrm{Zn}_{\mathrm{x}} \mathrm{S}\right)$ QDs to $\mathrm{ZnO}$ NPs should be discussed. The charge-transfer process between QDs and oxides is known as a charge-generation mechanism in QD photovoltaic devices ${ }^{41}$. For QLED, the QD-oxide junction is usually linked with single-dot emission intermittency (known as blinking), Auger recombination and reduction of photoluminescence ${ }^{42,43}$. In specific, n-type materials like $\mathrm{ITO}^{44}, \mathrm{TiO}_{\mathrm{x}}$ nanoparticles ${ }^{45,46}$ and p-type materials like $\mathrm{NiO}_{\mathrm{x}}$ nanoparticles ${ }^{47}$ can suppress the blinking of single dot at the expense of the photoluminescence quantum yield. Proposed blinking dynamics begin with electron (or hole) transfer from QD to n-type (or p-type) oxide (nanoparticles) and leave the QD in the positively (or negatively) charged off state; a back-transfer of charge is needed to turn the single dot on. For the case of QD-ZnO, reduction of photoluminescence was also observed and the model of electron transfer was proposed correspondingly 10,11 . As known, although the accurate value remains arguable, the electron affinity of blue $\left(\mathrm{Cd}_{1-\mathrm{x}} \mathrm{Zn}_{\mathrm{x}} \mathrm{S}\right) \mathrm{QDs}$ is significantly smaller than that of red QDs due to the increased bandgap energy and the small value of $m_{\mathrm{e}}^{*} / \mathrm{m}_{\mathrm{h}}^{*}$, wherein $m^{*}$ represents effective mass. The resultant CBM offset across the $\mathrm{QD}-\mathrm{ZnO}$ junction is reflected by the $J-F$ characteristics of electron-only devices in Fig. 1c. From these curves, although we cannot extract the barrier-height between $\mathrm{QD}$ and $\mathrm{ZnO}$ using models like Richardson-Schottkly transport ${ }^{48}$ and Arkhipov's theory ${ }^{49,50}$ which consider one metal-semiconductor junction, band diagram in Supplementary Fig. 6 suggests that $\mathrm{ZnS}$ at surface leads to substantial CBM offset which is enough to result in electrons transfer from blue dots to $\mathrm{ZnO}$. For the same reason, the back-transfer of electrons is not favored, eventually resulting in charge accumulation in $\mathrm{ZnO}$. Except for the offset of CBM energy, such charge transfer and trapping can be further facilitated by the structure and size of QDs. For example, the QDs with gradient-alloyed structure have a soft confinement of electrons which increases the chance of electron tunneling to the surface of QDs. Also, the blue QDs has a similar diameter to $\mathrm{ZnO}$ NPs $(\sim 5 \mathrm{~nm})$, therefore $\mathrm{ZnO}$ NPs can get closer proximity to blue 

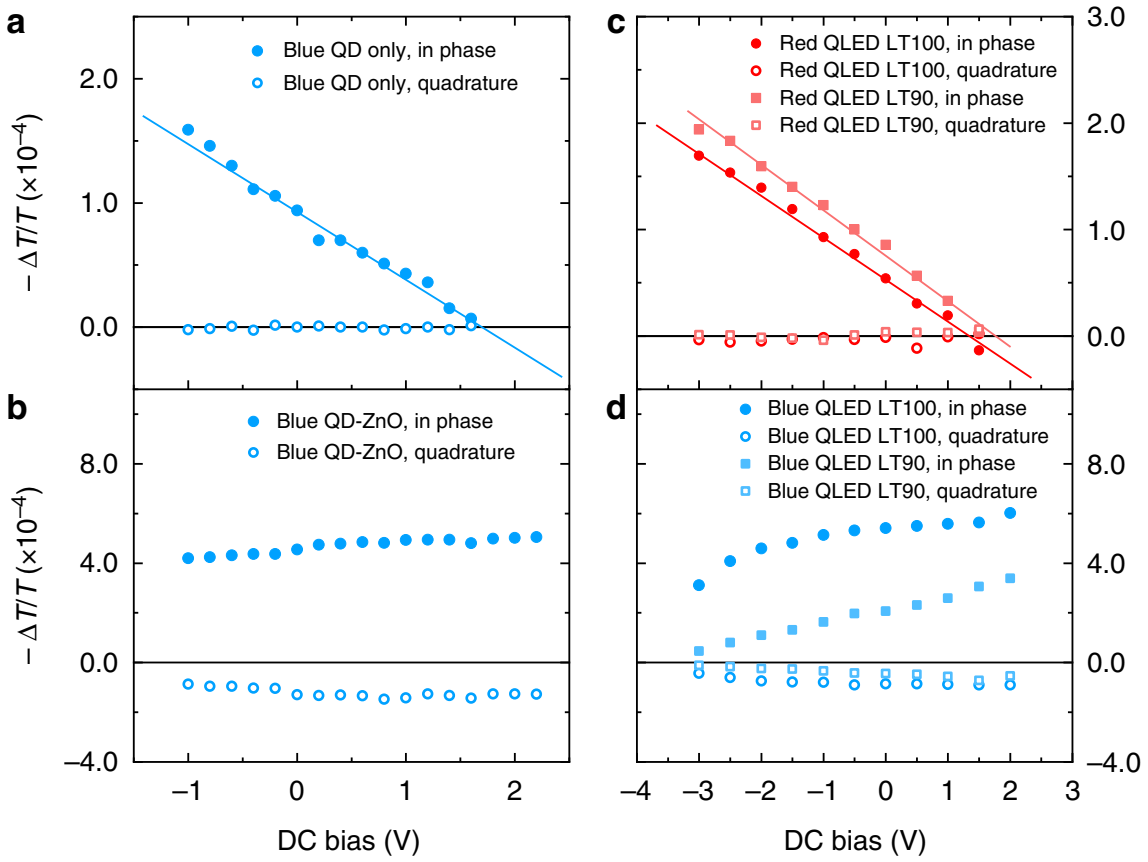

Fig. 5 The origin of charge modulation. a DC bias-dependent elecctro-absorption (EA) signal of the sample with a structure of ITO/blue (Cd $\left.d_{1-x} Z n_{x} S\right)$ QDs/ Al. $\mathbf{b}$ DC bias-dependent EA signal of the sample with a structure of ITO/blue $\left(\mathrm{Cd}_{1_{-}} \mathrm{Zn} \mathrm{n}_{\mathrm{x}} \mathrm{S}\right) \mathrm{QDs} / \mathrm{ZnO} / \mathrm{Al}$. $\mathbf{c}$ DC bias-dependent EA signal of a standard red device which is measured before and after the lifetime test. $\mathbf{d} D C$ bias-dependent EA signal of a standard blue device which is measured before and after the lifetime test. The probing wavelength is $570 \mathrm{~nm}$ for red $\left(\mathrm{Cd}_{1_{-}} \mathrm{Zn}_{x} \mathrm{Se}_{1_{-y}} \mathrm{~S}_{\mathrm{y}}\right)$ dots and $455 \mathrm{~nm}$ for blue $\left(\mathrm{Cd}_{1_{-x}} \mathrm{Zn}_{\mathrm{x}} \mathrm{S}\right)$ dots. The straight lines are indicators of the linear relationship between signal strength and applied bias

QDs and increase the chance of charge transfer. Therefore, we can describe the charge modulation effect as following. First, upon excitation of a blue dot, subsequent charge transfer leaves blue QDs positively charged and $\mathrm{ZnO}$ negatively charged, and their carrier concentration is modulated by the reference bias $\left(V_{\mathrm{ac}}\right)$. Then, the accepted electrons in $\mathrm{ZnO}$ experience trapping and de-trapping, which slows down the response of charges to the modulating bias and gives rise to the strong quadrature signals.

Through the spectra analysis (Fig. 4c, d) and the study of bias dependence (Fig. 5), we conclude that the insertion of a $\mathrm{ZnO}$ NPs layer results in charge transfer across the blue QD-ZnO interface as long as the blue QD is excited, resulting in strong chargemodulated signals. In the following, it shows that the charge transfer process is responsible for the voltage rise in ETL. Again, these effects are only observed in samples with both blue QDs and $\mathrm{ZnO}$. The consistence between $\mathrm{EA}$ and $C-V$ results should explain the different degradation mechanisms between red and blue QLED devices.

Charge balance. $C-V$ is an effective way to evaluate the charge balance in QLED. To minimize the contribution from (deep level) traps, a modulating frequency at $100 \mathrm{kHz}$ is used. We are able to obtain smooth curves even from heavily degraded devices. Under reversed bias, the whole device can be roughly treated as three planar capacitors connected in series, with each capacitor corresponding to the depleted HTL, QD layer and ETL ${ }^{51}$. When the applied bias turns to positive, a charge-unbalanced device will let the fast carrier inject first, i.e., electrons (holes) in red (blue) QLED, resulting in capacitance rise. The applied voltage concentrates on the transporting layer of the slow carrier, i.e., HTL (ETL) in red (blue) QLED, until the bias is high enough to cause efficient radiative recombination and capacitance drop. Given that both red and blue QLED are charge-unbalanced systems, measuring $C-V$ is an adequate method to evaluate the field distribution.
The $C-V$ results of standard red and blue QLED devices are plotted in the same semi-logarithmic scale. In Fig. 6a, the capacitance values of red QLEDs stay almost constant until the bias of $1.5 \mathrm{~V}$ and then decreases significantly after $2.5 \mathrm{~V}$. The relative constant value of $3.2 \mathrm{nF}$ roughly matches the geometric capacitance of HTL $(4.2 \mathrm{nF})$, QD layer $(>20 \mathrm{nF})$, and ETL $(\mathrm{ZnO}$, $25 \mathrm{nF}$ ) connected in series. In a red QLED device, electron injection is more efficient than that of holes, therefore the capacitance rise at $1.8 \mathrm{~V}$ means that the HTL (TFB) is the only layer remaining depleted and other layers have reached flat band and charge injection. In fact, the peak capacitance value of $4.0 \mathrm{nF}$ agrees with the geometric capacitance of a $40 \mathrm{~nm}$-thick TFB layer. The bias at which capacitance starts to decrease sharply marks the occurrence of efficient radiative recombination. A fresh red device has a luminance turn-on voltage of at $1.6 \mathrm{~V}$, however, reasonable electroluminescence efficiency $\left(\sim 15 \mathrm{~cd} \mathrm{~A}^{-1}\right)$ is not reached until a bias of $2.4 \mathrm{~V}^{12}$, which is consistent with our $C-V$ data. After the LT90 test (Fig. 5c), the red device experiences a slight increase of capacitance-transition voltage for about $0.1 \mathrm{~V}$ due to the same voltage rise in the HTL. Combining the result with that in Fig. 3a, we can conclude that slow degradation of red QLED originates from the mild degradation of HTL.

The $C-V$ results of blue QLED devices (TFB as HTL) are shown in Fig. 6b. Under reversed bias, the capacitance is almost the same as that of the red device due to similar device structure and materials. For the LT100 device, capacitance starts to increase at $2.8 \mathrm{~V}$, and the peak value reaches $5.2 \mathrm{nF}$. At a modulating frequency of $100 \mathrm{kHz}$, such an increase of capacitance is unlikely due to a depleted organic HTL. In fact, assuming the injection of holes is more efficient than that of electrons, the HTL in the blue device reaches the flat-band condition in prior to the ETL, leaving voltage concentrated across the ETL. This is consistent with the result of single-carrier devices (Fig. 1c and Supplementary Fig. 1c) and the results of EA measurements (Fig. 2). When the blue device degrades, the $C-V$ characteristics change significantly. In 


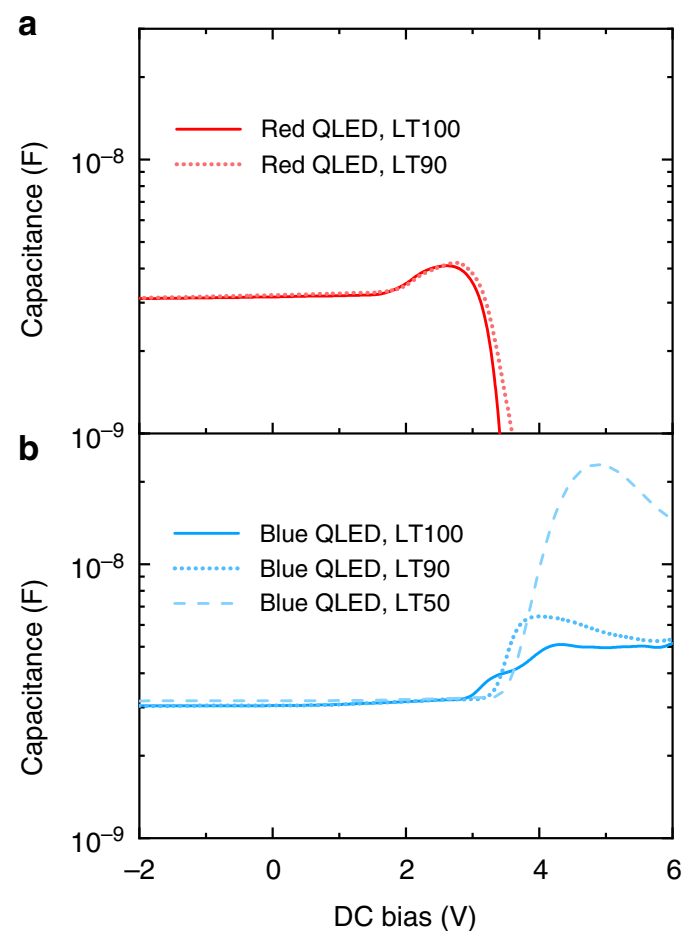

Fig. 6 Internal field distribution and charge balance. a The capacitancevoltage $(C-V)$ characteristics of a standard red device. $\mathbf{b}$ The $C-V$ characteristics of a standard blue device. In the measurements, the modulating frequency is $100 \mathrm{kHz}$, and the modulating amplitude is $20 \mathrm{mV}$. Each device is measured before and after the lifetime test

Fig. $6 \mathrm{~b}$, the capacitance of the LT90 sample turns on at $3.2 \mathrm{~V}$ and peaks at $6.6 \mathrm{nF}$. For the LT50 sample, the turn-on voltage and peak capacitance further increases to $3.3 \mathrm{~V}$ and $24 \mathrm{nF}$, respectively. The big shift of transition voltage is an evidence of significant charge accumulation due to inefficient charge transport, which is similar to the case of small-molecular OLED ${ }^{51,52}$. Further, the very high capacitance confirms that the injection holes is more efficient that electrons, and the charge accumulation occurs in the ETL. Here, with a bulk dielectric constant $(100 \mathrm{kHz})$ of $\varepsilon_{\mathrm{r}} \sim 30^{53}$, volume fraction of solids $\sim 0.7$, and a device area of $4 \mathrm{~mm}^{2}$, the geometric capacitance of a $30 \mathrm{~nm}$-thick $\mathrm{ZnO}$ NPs layer is roughly $25 \mathrm{nF}$, which is in good agreement with the peak capacitance of our LT50 sample. When the sample is biased to its peak capacitance, the $\mathrm{ZnO}$ ETL is the only functional layer that remains depleted. In blue QLED devices, the large barrier for electron injection and the charge transfer at the QD-ZnO interface result in significant charge accumulation during continuous driving, leading to increased voltage drop across the ETL and device degradation. For blue devices with PVK HTL (Supplementary Fig. 7), the carrier injection of holes and electrons are both inefficient, resulting in even field distribution. Since the degradation due to PVK is much faster than that due to ETL, the increase of capacitance is not very significant before the failure of device.

\section{Discussion}

To pinpoint the mechanism of device degradation in red and blue QLED, measurements of electro-absorption and capacitance-voltage characteristics are carried out. First, through the analysis of EA spectra, the emergence of $\mathrm{HTL}^{+}$was spotted in both red and blue devices after their lifetime tests, but the operating-voltage rise across the HTLs is insignificant. Therefore, HTL is responsible the slow degradation of red devices, however, it is not the culprit for the poor lifetime of blue devices. Next, evident by the results of electro-absorption and capacitance measurements, the voltage drop across the ETL $(\mathrm{ZnO})$ was found to increase significantly after the degradation of blue QLED devices. Upon the formation of QD-ZnO junction, $\mathrm{ZnO}$ NPs become the acceptor for the excited electrons in blue $\left(\mathrm{Cd}_{1-\mathrm{x}} \mathrm{Zn}_{\mathrm{x}} \mathrm{S}\right)$ QDs under the driving force of CBM offset, resulting in charge transfer and accumulation. Finally, capacitance-voltage characteristics of blue QLED confirm that the voltage consumption across the ETL increases significantly during the lifetime test. To solve the lifetime issue, it is necessary to develop ETL and QD materials that enable smaller CBM offset.

\section{Methods}

Preparation of materials. The blue $\left(\mathrm{Cd}_{1-\mathrm{x}} \mathrm{Zn}_{\mathrm{x}} \mathrm{S}\right)$, and red $\left(\mathrm{Cd}_{1-\mathrm{x}} \mathrm{Zn}_{\mathrm{x}} \mathrm{Se}_{1-\mathrm{y}} \mathrm{S}_{\mathrm{y}}\right)$ quantum dots used here were prepared according to methods reported previously in the literature, with appropriate modifications ${ }^{54,55}$. $\mathrm{ZnO}$ nanoparticles were synthesized using a solution-precipitation process as reported in the literature ${ }^{9}$. For a typical synthesis, a solution of zinc acetate in dimethyl sulphoxide (DMSO, $0.5 \mathrm{M}$ ) and $30 \mathrm{ml}$ of a solution of tetramethylammonium hydroxide (TMAH) in ethanol $(0.55 \mathrm{M})$ were mixed and stirred for $1 \mathrm{~h}$ in ambient air, then washed and dispersed in ethanol for device fabrication.

Device fabrication and characterization. Standard QLED devices were fabricated by spin coating on glass substrates that were commercially pre-coated with an indium-tin oxide anode (sheet resistance $\sim 25 \Omega \square^{-1}$ ). The substrates were cleaned consecutively in ultrasonic baths of deionized water, acetone and 2-propanol for 15 min each, and were then exposed to an ultraviolet ozone ambient for $15 \mathrm{~min}$. The substrates were spin-coated with PEDOT:PSS (AI 4083) and baked at $150^{\circ} \mathrm{C}$ for 15 min in air. The coated substrates were then transferred into a nitrogen-filled glove box for spin coating of layer of TFB, quantum dots and $\mathrm{ZnO}$ nanoparticles. TFB was purchased from American Dye Source, and used as supplied. The TFB layers were spin-coated at 4000 r.p.m. for $30 \mathrm{~s}$ using an $8 \mathrm{mg} \mathrm{ml}^{-1}$ solution in chlorobenzene, followed by baking at $150^{\circ} \mathrm{C}$ for $30 \mathrm{~min}$. Quantum dots and $\mathrm{ZnO}$ nanoparticle layers were then spin-coated and baked at $70^{\circ} \mathrm{C}$ for $30 \mathrm{~min}$. The optimized quantum-dot layer thicknesses were $\sim 16 \mathrm{~nm}$ for red $\left(15 \mathrm{mg} \mathrm{ml}^{-1}, 2500\right.$ r.p.m.), $\sim 20 \mathrm{~nm}$ for green $\left(18 \mathrm{mg} \mathrm{ml}^{-1}\right.$ quantum-dot solution, 2000 r.p.m. spin speed), and $\sim 20 \mathrm{~nm}$ for blue (18 $\mathrm{mg} \mathrm{ml}^{-1}, 2000$ r.p.m.), as determined from an efficiency comparison of devices with various quantum-dot layer thicknesses. Depending on the emitting wavelength, the thickness of the $\mathrm{ZnO}$ is controlled between 30 and $65 \mathrm{~nm}$ by changing the solution concentration and spin speed. Finally, the multilayer samples were loaded into a high-vacuum chamber (base pressure $\sim 1 \times 10^{-7}$ Torr) for deposition of an Al cathode $(100 \mathrm{~nm})$, patterned by a shadow mask to form devices with an active area of $4 \mathrm{~mm}^{2}$. All devices were encapsulated in commercially available ultraviolet-curing epoxy and cover glass. For the lifetime test, the encapsulated samples were measured under ambient conditions using a commercialized lifetime test system (Guangzhou New Vision Opto-electronic Technology Co. Ltd.).

Electro-absorption. The samples were kept inside a cryostat (Janis VPF-100, liquid nitrogen) with a pressure of $10^{-3}$ Torr. A monochromatic parallel beam probes the sample through the ITO side with an incident angle of 45 and is reflected by the back electrode. To modulate the internal electric field for low-noise detection, a sinusoidal voltage with frequency of $1 \mathrm{~K} \mathrm{~Hz}$ was superimposed to the DC bias, producing a periodical bias in the form of $V(t)=V_{\mathrm{dc}}+V_{\mathrm{ac}} \sin \omega t$, wherein $\omega$ is the modulating frequency. When measuring the electro-absorption spectra, the DC bias is negative to avoid carrier injection into the devices. The back-reflected signal got further absorbed by the organic layer before being collected by the photodetectors. Calibrated silicon and germanium photodetectors were used to detect the reflected signal. A current amplifier and a lock-in amplifier (SR830) were connected to the detector and locked to 1st harmonic frequency for low-noise measurement. The final signal was the ratio of the signals with and without the modulation of $V_{\mathrm{ac}}$. To lower the noise, the time constant of the lock-in was set at $1 \mathrm{~s}$. Each data point is averaged from 16 measurements.

Capacitance-voltage characterization. Measurements were carried out with an Agilent $4282 \mathrm{~A}$ precision LCR meter and the data was automatically acquired by a computer. To avoid the effect of trapped charges, the modulating frequency and the modulating amplitude is $100 \mathrm{kHz}$ and $20 \mathrm{mV}$, respectively.

\section{Data availability}

Source data are available from corresponding authors upon request.

Received: 16 April 2018 Accepted: 24 January 2019

Published online: 15 February 2019 


\section{References}

1. Shirasaki, Y., Supran, G. J., Bawendi, M. G. \& Bulović, V. Emergence of colloidal quantum-dot light-emitting technologies. Nat. Photon. 7, 13-23 (2013).

2. Supran, G. J. et al. QLEDs for displays and solid-state lighting. Mrs. Bull. 38, 703-711 (2013).

3. Dai, X., Deng, Y., Peng, X. \& Jin, Y. Quantum-dot light-emitting diodes for large-area displays: towards the dawn of commercialization. Adv. Mater. 29, 1607022 (2017).

4. Murray, C. B., Norris, D. J. \& Bawendi, M. G. Synthesis and characterization of nearly monodisperse $\mathrm{CdE}$ ( $\mathrm{E}=$ sulfur, selenium, tellurium) semiconductor nanocrystallites. J. Am. Chem. Soc. 115, 8706-8715 (1993).

5. Peng, X. et al. Shape control of CdSe nanocrystals. Nature 404, 59-61 (2000).

6. Peng, Z. A. \& Peng, X. Formation of high-quality CdTe, CdSe, and CdS nanocrystals using CdO as precursor. J. Am. Chem. Soc. 123, 183-184 (2001).

7. Chen, O. et al. Compact high-quality CdSe-CdS core-shell nanocrystals with narrow emission linewidths and suppressed blinking. Nat. Mater. 12, 445-451 (2013).

8. Caruge, J. M., Halpert, J. E., Wood, V., Bulović, V. \& Bawendi, M. G. Colloidal quantum-dot light-emitting diodes with metal-oxide charge transport layers. Nat. Photon. 2, 247-250 (2008)

9. Qian, L., Zheng, Y., Xue, J. \& Holloway, P. H. Stable and efficient quantumdot light-emitting diodes based on solution-processed multilayer structures. Nat. Photon. 5, 543-548 (2011)

10. Mashford, B. S. et al. High-efficiency quantum-dot light-emitting devices with enhanced charge injection. Nat. Photon. 7, 407-412 (2013).

11. Dai, X. et al. Solution-processed, high-performance light-emitting diodes based on quantum dots. Nature 515, 96-99 (2014)

12. Yang, Y. et al. High-efficiency light-emitting devices based on quantum dots with tailored nanostructures. Nat. Photon. 9, 259-266 (2015).

13. Kovalenko, M. V., Protesescu, L. \& Bodnarchuk, M. I. Properties and potential optoelectronic applications of lead halide perovskite nanocrystals. Science 358, 745-750 (2017).

14. Qian, L. et al. 6-2: Invited Paper: Key challenges towards the commercialization of quantum-dot light-emitting diodes. SID Symp. Dig. Tech. Pap. 48, 55-57 (2017).

15. Cao, W. et al. Highly stable QLEDs with improved hole injection via quantum dot structure tailoring. Nat. Commun. 9, 2608 (2018).

16. Shen, H. et al. Efficient and long-lifetime full-color light-emitting diodes using high luminescence quantum yield thick-shell quantum dots. Nanoscale $\mathbf{9}$, 13583-13591 (2017)

17. Levermore, P. et al. 38-1: Invited Paper: Ink-jet-printed OLEDs for display applications. SID Symp. Dig. Tech. Pap. 47, 484-486 (2016).

18. Yamada, T. et al. 57-2: Invited Paper: Latest development of high-performance OLED material suitable for printing. SID Symp. Dig. Tech. Pap. 48, 845-848 (2017)

19. Cho, K.-S. et al. High-performance crosslinked colloidal quantum-dot lightemitting diodes. Nat. Photon. 3, 341 (2009).

20. Wood, V. et al. Selection of metal oxide charge transport layers for colloidal quantum dot LEDs. ACS Nano 3, 3581-3586 (2009).

21. Hüfner, S. Photoelectron Spectroscopy: Principles and Applications (Springer Science \& Business Media, 2013)

22. Lee, D.-H., Liu, Y.-P., Lee, K.-H., Chae, H. \& Cho, S. M. Effect of hole transporting materials in phosphorescent white polymer light-emitting diodes. Org. Electron. 11, 427-433 (2010).

23. Wang, O. et al. High-efficiency, deep blue $\mathrm{ZnCdS} / \mathrm{Cd}_{\mathrm{x}} \mathrm{Zn}_{1-\mathrm{x}} \mathrm{S} / \mathrm{ZnS}$ quantumdot-light-emitting devices with an EQE exceeding 18\%. Nanoscale 10 5650-5657 (2018).

24. Kondakov, D. Y., Lenhart, W. C. \& Nichols, W. F. Operational degradation of organic light-emitting diodes: mechanism and identification of chemical products. J. Appl. Phys. 101, 024512 (2007)

25. Lee, K.-H. et al. Highly efficient, color-pure, color-stable blue quantum dot light-emitting devices. ACS nano 7, 7295-7302 (2013).

26. Kwak, J. et al. Bright and efficient full-color colloidal quantum dot light-emitting diodes using an inverted device structure. Nano. Lett. 12, 2362-2366 (2012).

27. Shen, H. et al. High-efficiency, low turn-on voltage blue-violet quantum-dotbased light-emitting diodes. Nano. Lett. 15, 1211-1216 (2015).

28. Anikeeva, P. O., Halpert, J. E., Bawendi, M. G. \& Bulović, V. Quantum dot light-emitting devices with electroluminescence tunable over the entire visible spectrum. Nano. Lett. 9, 2532-2536 (2009).

29. Lane, P. A., Chen, S. \& So, F. Electromodulated doping of the hole transport layer in a small molecule organic light-emitting diode. J. Photon Energy 1, 011020 (2011)

30. So, F. \& Kondakov, D. Degradation mechanisms in small-molecule and polymer organic light-emitting diodes. Adv. Mater. 22, 3762-3777 (2010).

31. Sebastian, L. \& Weiser, G. One-dimensional wide energy bands in a polydiacetylene revealed by electroreflectance. Phys. Rev. Lett. 46, 1156-1159 (1981).
32. Tsang, S. W., Chen, S. \& So, F. Energy level alignment and sub-bandgap charge generation in polymer: fullerene bulk heterojunction solar cells. Adv. Mater. 25, 2434-2439 (2013).

33. Chen, S., Tsang, S. W., Lai, T. H., Reynolds, J. R. \& So, F. Dielectric effect on the photovoltage loss in organic photovoltaic cells. Adv. Mater. 26, 6125-6131 (2014).

34. Campbell, I. H. et al. Measuring internal electric fields in organic lightemitting diodes using electroabsorption spectroscopy. Polym. Adv. Technol. 8, 417-423 (1997).

35. Lany, S. \& Zunger, A. Anion vacancies as a source of persistent photoconductivity in II-VI and chalcopyrite semiconductors. Phys. Rev. B 72 035215 (2005).

36. Liu, X., Iimori, T., Ohshima, R., Nakabayashi, T. \& Ohta, N. Electroabsorption spectra of PbSe nanocrystal quantum dots. Appl. Phys. Lett. 98, 161911 (2011).

37. Awasthi, K., Iimori, T. \& Ohta, N. Integral method analysis of electroabsorption spectra and its application to quantum dots of PbSe. J. Phys. Chem. C. 118, 18170-18176 (2014).

38. Wehrenberg, B. L. \& Guyot-Sionnest, P. Electron and hole injection in PbSe quantum dot films. J. Am. Chem. Soc. 125, 7806-7807 (2003).

39. Sargent, E. H. Infrared quantum dots. Adv. Mater. 17, 515-522 (2005).

40. Colvin, V. L., Cunningham, K. L. \& Alivisatos, A. P. Electric field modulation studies of optical absorption in CdSe nanocrystals: dipolar character of the excited state. J. Chem. Phys. 101, 7122-7138 (1994)

41. Kamat, P. V. Quantum dot solar cells. Semiconductor nanocrystals as light harvesters. I. Phys. Chem. C. 112, 18737-18753 (2008).

42. Galland, C. et al. Two types of luminescence blinking revealed by spectroelectrochemistry of single quantum dots. Nature 479, 203-207 (2011)

43. Efros, A. L. \& Nesbitt, D. J. Origin and control of blinking in quantum dots Nat. Nanotechnol. 11, 661-671 (2016).

44. Jin, S., Song, N. \& Lian, T. Suppressed blinking dynamics of single QDs on ITO. ACS nano 4, 1545-1552 (2010).

45. Jin, S. \& Lian, T. Electron transfer dynamics from single CdSe/ZnS quantum dots to $\mathrm{TiO}_{2}$ nanoparticles. Nano. Lett. 9, 2448-2454 (2009)

46. Hamada, M., Nakanishi, S., Itoh, T., Ishikawa, M. \& Biju, V. Blinking suppression in $\mathrm{CdSe} / \mathrm{ZnS}$ single quantum dots by $\mathrm{TiO}_{2}$ nanoparticles. ACS nano 4, 4445-4454 (2010).

47. $\mathrm{Wu}, \mathrm{X}$. \& Yeow, E. K. Charge-transfer processes in single CdSe/ZnS quantum dots with p-type NiO nanoparticles. Chem. Commun. 46, 4390-4392 (2010).

48. Sze, S. M. Semiconductor Devices: Physics and Technology (John Wiley \& Sons, Hoboken, 2008)

49. Arkhipov, V., Emelianova, E., Tak, Y. \& Bässler, H. Charge injection into light emitting diodes: Theory and experiment. J. Appl. Phys. 84, 848-856 (1998).

50. Wolf, U., Arkhipov, V. I. \& Bässler, H. Current injection from a metal to a disordered hopping system. I. Monte Carlo simulation. Phys. Rev. B 59, 7507-7513 (1999)

51. Kondakov, D. Y., Sandifer, J. R., Tang, C. W. \& Young, R. H. Nonradiative recombination centers and electrical aging of organic light-emitting diodes: direct connection between accumulation of trapped charge and luminance loss. J. Appl. Phys. 93, 1108-1119 (2003).

52. Chen, S., Jiang, X. \& So, F. Hole injection polymer effect on degradation of organic light-emitting diodes. Org. Electron. 14, 2518-2522 (2013).

53. Langton, N. \& Matthews, D. The dielectric constant of zinc oxide over a range of frequencies. Br. J. Appl. Phys. 9, 453-456 (1958).

54. Bae, W. K., Char, K., Hur, H. \& Lee, S. Single-step synthesis of quantum dots with chemical composition gradients. Chem. Mater. 20, 531-539 (2008).

55. Bae, W. K., Nam, M. K., Char, K. \& Lee, S. Gram-scale one-pot synthesis of highly luminescent blue emitting $\mathrm{Cd}_{1-} \mathrm{Zn}_{\mathrm{x}} \mathrm{S} / \mathrm{ZnS}$ nanocrystals. Chem. Mater. 20, 5307-5313 (2008)

\section{Acknowledgements}

The work is supported by National Natural Science Foundation of China (Grant No. 61804102), Natural Science Foundation of Jiangsu Province (Grant No. BK20180835), and "National Thousand Youth Talents Plan".

\section{Author contributions}

S.C. conceived the idea and conducted the measurement of electro-absorption and capacitance-voltage characteristics. S.C. and L.Q. designed the experiments. W.C. con tributed to the data collection and analysis for $L-J-V$ characteristics, QLED lifetime, and single-carrier devices; T.L. assisted the measurement of electro-absorption and capacitance-voltage characteristics. Y.Y. contributed to material synthesis; S.C. wrote the first draft of the manuscript; W.C., S.T., Y.Y., X.Y., and L.Q. provided suggestions for revisions. L.Q. and X.Y. supervised the QLED research at TCL. S.C. and W.C. contributed equally to this work. 


\section{Additional information}

Supplementary Information accompanies this paper at https://doi.org/10.1038/s41467019-08749-2.

Competing interests: The authors declare no competing interests.

Reprints and permission information is available online at http://npg.nature.com/ reprintsandpermissions/

Journal peer review information: Nature Communications thanks the anonymous reviewers for their contribution to the peer review of this work.

Publisher's note: Springer Nature remains neutral with regard to jurisdictional claims in published maps and institutional affiliations. (c) (i) Open Access This article is licensed under a Creative Commons Attribution 4.0 International License, which permits use, sharing, adaptation, distribution and reproduction in any medium or format, as long as you give appropriate credit to the original author(s) and the source, provide a link to the Creative Commons license, and indicate if changes were made. The images or other third party material in this article are included in the article's Creative Commons license, unless indicated otherwise in a credit line to the material. If material is not included in the article's Creative Commons license and your intended use is not permitted by statutory regulation or exceeds the permitted use, you will need to obtain permission directly from the copyright holder. To view a copy of this license, visit http://creativecommons.org/ licenses/by/4.0/.

(C) The Author(s) 2019 